\title{
Response and compliance of botulinum toxin with or without oral treatment in hemifacial spasm
}

\author{
Dilip Maheshwari ${ }^{1}$, Bharat Bhushan ${ }^{2 *}$, Vijay Sardana ${ }^{3}$ \\ ${ }^{1}$ Associate Professro, ${ }^{2}$ Professor, ${ }^{3}$ Senior Professor, ${ }^{1-3}$ Dept. of Neurology, ${ }^{1-3}$ Govt. Medical College, Kota, Rajasthan, India
}

*Corresponding Author: Dilip Maheshwari

Email: drbhushan90@yahoo.com

\begin{abstract}
Hemifacial spasm (HFS) is the commonest presentation than any other movement disorder in Botulinum Toxin (BoNT) therapy clinic. Objective: To evaluate response and compliance of BoNT in HFS. To observe the BoNT duration of therapy effect with or without oral treatment in HFS.

Materials and Methods: This is a retrospectively carried out study at government medical college, Kota, Rajasthan. Seventy eight patients of HFS were evaluated out of 142 cases from movement disorder clinic who received BoNT therapy from December 2012 to January 2019. Patients were eligible if they were older than 18 years and clinically confirmed diagnosis of HFS.

Results: Mean age was $48.5 \pm 4.32$ years. Forty seven (60.25\%) were female. Left hemifacial spasm was seen in $71.7 \%$. Seventy percent of patients were having an initial response with oral medication. Total numbers of injections were 304 and average applications per patient were 3.89. The average dose per patient given was $22 \pm 3.2$ units. Latency and duration of effect of BoNT therapy were $9 \pm 2$ days and 4.3 months respectively. Mean interval between injection and average follow up was 5.3 and 12.6 months respectively. The overall success rate was $94.78 \%$. Adverse effect on the total application was $11.68 \%$. Most common adverse effect was ptosis and average recovery period of all adverse effect was $9 \pm 5$ days. One patient developed vaso-vagal syncope which recovered immediately. Comparision of BoNT therapy with continued oral treatment group and BoNT therapy alone was insignificant $(\mathrm{P}=0.925)$; however the duration of effect was longer with combined treatment than BoNT therapy alone.

Conclusion: We suggest that response of treatment in HFS with BoNT along with oral medication support may be more effective as compared to BoNT alone however large study trials are to be needed. Compliance to BoNT therapy depends upon side effect profile and pharmacoeconomic burden.
\end{abstract}

Keywords: Hemifacial spasm, Botulinum toxin, Response, Compliance.

\section{Introduction}

Hemifacial spasm (HFS) is characterized by brief or persistent involuntary paroxysmal contractions of the muscles innervated by facial nerve that are typically unilateral and may occasionally occur bilaterally. It is peripherally induced movement disorder and usually starts in the orbicularis oculi muscle and spread to other adjacent muscles like frontalis and zygomaticus major ipsilaterally. Mostly it is an idiopathic disorder but sometimes may be secondary to posterior fossa pathology leading to peripheral dysfunction of facial nerve. So Radiological imaging is not important for the diagnosis but it may be worthwhile to exlude the rare but serious conditions like facial nerve compression (arteriovenous malformations, tumour, aneurysm) or pontine demyelination., ${ }^{1,2,3}$

Hemifacial spasm affects life of patient cosmetically as well as sleep onset disturbance due to its persistence, though HFS is not life threatening. Mostly patient requires lifelong treatment which affects patient's life professionally and functionally. HFS affects women more than men and it usually appears in the fourth to seventh decade of life $^{4}$. At present available treatment are semi invasive in the form of botulinum neurotoxin (BoNT) or major surgical decompression procedure.

The effect of the toxin is reversible and temporary so repeated injections are needed. ${ }^{7}$ There are limited data to analyse the long term efficacy, compliance and reversibility of side effect profile in Indian population. The objective of current study is to evaluate six years experience of tertiary care centre for looking at response and compliance of BoNT with or without oral treatment in Hemifacial spasm.

\section{Materials and Methods}

This study was retrospectively carried out at Neurology department, government medical college, Kota, Rajasthan. One forty two patients were retrospectively scrutinized from movement disorder clinic who received BoNTfrom December 2012 to January 2019. Out of 142 patients, 78 cases of HFS were analyzed for the response, side effects reversibility and compliance to BoNT. Patients older than 18 years and clinically confirmed diagnosis of HFS with signed written consent were eligible for this study. Only those patients were taken who had regular follow up and received at least one botollinium toxin injection. Data were observed before injection, second and third month after each treatment as per record available. Repeat injection was based on the patient need for treatment. The response was evaluated during follow up and telephonically by a set of questionnaire. Visual analogue scale (VAS) scale was used to record the response of BoNT. The response was defined as at least a significant improvement according to both the patient and objective impression recorded by using a VAS ranging from $0 \%$ to $100 \%$. The study was approved by; the institutional ethical committee. Patient with pregnancy and with known contraindication to BoNT were excluded.

Various variables were assessed and analysed from record; including average time, period of symptoms onset to oral treatment before receiving BoNT therapy, total number 
of treatments, average dose per patient, average applications per patient, mean latency of effect (It is the time interval between the injection and the first sign of improvement after treatment), average duration of effect (time interval between the first clinical effect and the moment patient felt the need of retreatment), subjective scale evaluation (VAS), total patients having adverse effects, total number of adverse effects on total applications, mean interval between injections, mean follow up and success rate per injection. Reversibility period of side effects was recorded after each injection session.

Compliance was categorised as a good, poor and non compliant group. Good compliance was defined when the patient received three or more doses, the time interval between onset of recurrent symptom and reinjection is less than one month. Poor compliance was notified when the patient received two or fewer doses, the time interval between onset of recurrent symptom and reinjection is more than one month and along with oral medications received. Patients who received an only single session of therapy and continued oral medication were categorised as a non compliant group.

\section{Statistical Analysis}

Mean, median, average and percentage were calculated where it is applicable. Standard deviation and Chi Square test was used to compare duration of effect of BoNT with or without oral medication. A $\mathrm{P}$ value of less than 0.05 was set for statistical significance.

\section{Results}

Total 142 cases received BoNT therapy in movement disorder clinic, of which $78(54.93 \%)$ cases of HFS, 22(15.49\%) Blephrospasm, 15(10.56\%) cervical dystonia, $11(07.74 \%)$ writers cramps, $9(6.33 \%)$ oromandibular dystonia, $5(3.52 \%)$ spasticity and $2(1.40 \%)$ migraine. (Table 1)

Seventy eight patients of HFS were evaluated retrospectively. Mean patient age was 48.5 years with SD \pm 4.32 years. Forty seven $(60.25 \%)$ were female. Fifty six $(71.7 \%)$ had left and $22(28.20 \%)$ had right hemifacial spasm. Preinjection oral treatment was received by all patients. Seventy percent patients were having initial response with oral medication on subjective analysis and average time period of symptoms onset to oral treatment was 3.24 months. Total numbers of applications given for HFS were 304. Average dose per patient given was $22 \pm 3.2$ units. An average application per patient was 3.89. Mean onset latency of BoNT therapy effect was $9 \pm 2$ days. The average duration of effect was 4.3 months. On a subjective scale of $0-100 \%$ improvement, the effect of BoNT therapy was $82.37 \pm 4.3 \%$. Mean interval between injections was 5.3 months (1.9SD). Mean Follow up was 12.6 months. The overall success rate was $94.78 \%$ of all the injection session (Table 2). Eight patients $(10.25 \%)$ had a reduction of dose in the apprehension of side effects and 2 patients had an increase in dose and remaining patients received similar doses on follow up. None of the patients observed to have resistance.

Eight $(10.2 \%)$ patients developed adverse effects while an adverse effect on total application was 36(11.68\%). Most common adverse effect was ptosis after 8 applications followed by itching, facial paresis, conjuctival congestion. The average recovery period was $9 \pm 5$ days. The maximum recovery period was seen in diplopia (14 \pm 6 days) followed by ptosis and facial paresis. One patient developed vasovagal syncope which recovered immediately and was unrelated to BoNT. There was no report of systemic or severe adverse effects. (Table 3 )

Minimal preceding duration of oral treatment before receiving BoNT therapy was less than 2 months in 3 patients while it was more than 6 months in 36 patients and remaining were in between. Comparision of BoNT therapy with oral treatment arm and BoNT therapy alone was statistically insignificant $(\mathrm{P}=0-925)$ in all categories however, duration of effect was longer in BoNT therapy with oral category than BoNT therapy alone. (Table 4)

Good compliance was observed in 26 patients but 18 patients were noncompliant for BoNT therapy due to financial constraint, delayed recovery period of side effects, subjective tolerability of HFS with oral treatment and lost to follow up from present centre. (Table 5).

\section{Discussion}

This retrospective study is intended to evaluate the effect of BoNT therapy alone versus continued oral support by default to the treatment of HFS. BoNT therapy has emerged as an effective treatment for numerous hyperactive movement disorders. Evidence support a level B recommendation for BoNT on Class II studies because of its efficacy and safety profile. ${ }^{5}$ BoNT causes flaccid paralysis by blocking the release of acetylcholine at the neuromuscular junction. BoNT is taken up by the nerve cells at the neuromuscular junction, and damages proteins within the nerve cell that are needed for active functioning of synaptic vesicles to release acetylcholine. ${ }^{6}$ So BoNT is currently considered as gold standard for treatment of HFS as it is minimally invasive and well tolerated therapy. BoNT therapy is probably the second most important discovery in movement disorders therapy after levodopa. Our study demonstrated response and compliance of BoNT therapy in HFS. Response to the treatment was similar to the earlier studies published in the various literature. ${ }^{5,6,7}$ Success rate per injection in the present study was $94.78 \%$ which was comparable with a study done by J P M Batistti et al. ${ }^{8}$ and Pandey $S$ et al. ${ }^{9}$ this reflects a high degree of patient satisfaction in the present study. Female preponderance with left sided lateralization was similar to other studies. Response to therapy was influenced by combined oral treatment along with BoNT which was more favourable than BoNT alone, this is contrary to Barbosa et al. ${ }^{10}$, who reported that use of oral medication was highly unsatisfactory but did not interfere with response to BoNT treatment. Response to therapy was influenced by preceding duration of illness. The patients having a long duration of 
illness preceding BoNT therapy were having a shorter response as compared to short duration illness. Mean latency of effect to appear was $9 \pm 2$ days while other studies showing lower (7.1 days) and some was having more mean latency (11.63days). ${ }^{11-13}$

In the present study the average duration of effect was 4.3 months which was higher than the study performed by Batisti et al. ${ }^{8}$ Barbosa et al. ${ }^{10}$ and defazzio et al. ${ }^{14}$ Possible cause of longer duration was a continuation of oral treatment along with BoNT therapy. Although there was a similar duration of effect between first and last BoNT injection it endorsed the previous study ${ }^{10,14}$. There was no significant increment and decrement in the doses of BoNT from first to last application which was not in agreement with Barbosa et $\mathrm{al}^{9}$ and Hsuing et al. ${ }^{15}$

The present study reveals all side effects were reversible with a variable recovery period. Diplopia recovered with the longest duration ( $14 \pm 6$ days) among all side effects observed. One patient developed vaso-vagal syncope during BoNT application which was not reported earlier. So we would like to suggest extreme neck movements should be avoided in patient with a background history of vaso-vagal syncope and procedure should be carried out in the settings of readily available emergency care. Most common side effect was ptosis similar to Rieders et al. ${ }^{16}$ Recovery period for ptosis in our patient was $11 \pm 7$ days. Four patient discontinued treatment from our clinic due to receiving treatment from another centre.

This was a retrospective study so it has inherent problems related to treatment and compliance in the study but no potential bias. Second limitation was that small number of patients enrolled in the present study so statistical significance of combined therapy oral with BoNT was not powerful enough to detect differences. Third limitation was that we have not included patients with other type of movement disorder beside hemifacial spasm and the result found cannot be directly extrapolated to other conditions. Finally because of the short-term follow up and retrospective study of the patients we have not addressed other important areas related to BoNT therapy response and compliance in movement disorders.

In conclusion despite the limitations of this study finding of present study suggest that treatment of HFS with BoNT therapy along with oral medication support may be more effective as compared to BoNT therapy alone. Compliance to treatment depends upon magnitude of side effects and pharmacoeconomic burden. However, future larger prospective studies are needed to explore further valuable significance of BoNT therapy along with oral medication support.

\section{Conflict of Interest: None.}

\section{References}

1. Colosimo $\mathrm{C}$ et al. A comparative study of primary and secondary Hemifacial spasm. Arch Neurol 2006;63:441-44.

2. Mastura N, Kondo A. Trigeminal neuralgia and Hemifacial spasm as false localizing signs in patients with a contralateral mass of the posterior cranial fossa. J Neurosurg 1996;84:106771.

3. Nagata S, Matsushima T, Fujii K, Masashi F, kuromatsu C. Hemifacial spasm due to tumour, aneurysm, or arteriovenous malformation. Surg Neurol 1992;38:204-9

4. Auger 1990, Auger RG, Whisnant JP. Hemifacial spasm in Rochester and Olmested Country, Minnesota, 1960 to 1984. Arcieves Neurol 1990;47(11):1233-4

5. Hallett M. Evidence-based review and assessment of botulinum toxin for the treatment of movement disorders. Toxicon 2013;67:94-114.

6. Brin. Scientific and Therapeutic Aspects of Botulinum Toxin. Philadelphia: Lippincott Williams \& Wilkins,2002.

7. Xu YP, Shen YP Zhu QB, Gu J, Lin SZ. Efficacy of botulinum toxin A for treatment of unilateral spasms of eyelid and its prognosis. Eur Med Pharmacol Sci 2013;17(21):2974-9.

8. Batisti M, Kleinfelder F, Galli NB, Moro A, Munhoz RP, Teive HA et al. Treatment of hemifacial spasm with botulinum toxin type a: effective, long lasting and well tolerated. Arq Neuropsiquiatr 2017;75(2):87-91.

9. Pandey S, Jain S. Clinical feature and response to botulinum toxin in primary and secondary hemifacial spasm. Neurol India 2018;66:1036-42.

10. Barbosa ER, Takada LT, Goncalves LR, Costa RM, SilveiraMoriyama L, Chien HF. Botulinum toxin type a in the treatment of hemifacial spasm: an 11- year experience. Arq Neuropsiquiatr 2010;68(4):502-5.

11. Thussu A, Barman C, Prabhakar S. Botulinum toxin in treatment of hemifacial spasm and blephrospasm: objective response evaluation. Neurol India 1999;47(3): 206-9.

12. Bares M, Streitova H. Longterm therapy of benign essential blephrospasm and facial hemispasm with botulinum toxin A: Retrospective assessment of the clinical and quality of life impact in patient treated for more than 15 years. Acta Neurol Belg 2014;45:1-7.

13. Felicio AC, Godeiro Junior CO, Borgess V, Silva S, Ferraz HB. Clinical assessment of patients with primary and post paralytic hemifacial spasm: A retrospective study. Arq Neuropsiquiatr 2007;65(3B):783-6.

14. Defazio G, Abbruzzese G, Girlanda P, et al. Botulinum toxin A treatment for primary hemifacial spam. Arch Neurol 2002;59:418-20.

15. Huising GYR, Das SK, Ranawaya R, Lafontain AL, Suchowersky O. Long-term efficacy of botulinum toxain A in treatment of various movement disorder over a 10 -year period. Movt Disord 2002;17(6):1288-93.

16. Rieder CRM, Schestatsky P, Socal MP, Monte TL, Fricke D, Costa J. A double-blind, randomized, crossover study of prosigne versus botox in patients with blephrospasm and hemifacial spasm. Clin Neuropharmacol 2007;30(1):39-42.

How to cite this article: Maheshwari D, Bhushan B, Sardana $\mathrm{V}$. Response and compliance of botulinum toxin with or without oral treatment in hemifacial spasm. India J Neurosci 2019;5(2):50-52. 